\title{
Precision Measurement of the Accumulator Beam Energy
}

\author{
Steven J. Werkema
}

February 28,2000

\section{Introduction}

The Antiproton Source Accumulator has been used by Fermilab experiments E760 and E835 to search for and measure the various states of charmonium below the open charm threshold. Accurate determination of the resonance parameters (mass, width, and branching ratios) of these states requires a precise measurement of the antiproton beam energy. The purpose of this report is to give a detailed description of the method that is used to accomplish a precision measurement of the antiproton beam energy.

The basic strategy is to determine the energy of the beam by a measurement of its velocity. The energy, $E$, of a particle, written in terms of its velocity parameter, $\beta$, is:

$$
E=\frac{m c^{2}}{\sqrt{1-\beta^{2}}}
$$

$m$ is the mass of an Antiproton. The velocity of a circulating antiproton is the product of its revolution frequency and the length of its orbit, viz. $c \beta=f_{\text {rev }} L$. Writing (1) in terms of $f_{r e v}$ and $L$ gives:

$$
E\left(f_{r e v}, L\right)=\frac{m c^{2}}{\sqrt{1-\left(\frac{f_{r e v} L}{c}\right)^{2}}}
$$

Therefore, the average velocity of the beam, and hence its energy, can be obtained by a measurement of the beam's average revolution frequency and orbit length.

It is apparent from equations (1) and (2) that as the beam velocity approaches the speed of light the precision of this measurement rapidly degrades. Differentiating (2) with respect to $f_{\text {rev }}$ and $L$ gives the uncertainty in the measurement of the beam energy, $\delta E$, as:

$$
\frac{\delta E}{E}=\beta^{2} \gamma^{2}\left(\frac{\delta f_{r e v}}{f_{\text {rev }}}+\frac{\delta L}{L}\right)
$$

Thus, the relative uncertainty in this measurement of the beam energy grows as $\gamma^{2}$ with increasing energy.

The quantity of interest to the experiments using the Antiproton Source is the energy in the center of mass reference frame. The center of mass energy, $E_{c m}$, is related to the laboratory frame energy, $E$, by:

$$
E_{c m}=\sqrt{2} m c^{2} \sqrt{1+\frac{E}{m c^{2}}}
$$

The uncertainty in the measurement of $E_{c m}$ is: 


$$
\begin{aligned}
\frac{\delta E_{c m}}{E_{c m}} & =\frac{\beta^{2} \gamma^{3}}{2(1+\gamma)}\left(\frac{\delta f_{r e v}}{f_{r e v}}+\frac{\delta L}{L}\right) \\
& =\gamma\left(\frac{p c}{E_{c m}}\right)^{2}\left(\frac{\delta f_{r e v}}{f_{\text {rev }}}+\frac{\delta L}{L}\right)
\end{aligned}
$$

Table I gives the uncertainties in $E$ and $E_{c m}$ at various energies of interest to experiments E760 and E835 (Charmonium spectroscopy). The values in Table I are calculated from equations (3) and (5).

Table I. Uncertainty in Beam Energy Measurement

\begin{tabular}{|c|c|c|c|}
\hline Energy: & $\begin{array}{c}\mathbf{8 ~ G e V} \\
\text { Stacking Energy }\end{array}$ & $\begin{array}{c}\psi^{\prime} \text { Resonance } \\
\boldsymbol{E}_{c m}=\mathbf{3 6 8 6 . 0 0 0 ~ M e V} \\
\mathbf{E}=\mathbf{6 3 0 1 . 9 4 8 ~ M e V}\end{array}$ & $\begin{array}{c}\mathbf{J} / \psi \text { Resonance } \\
\boldsymbol{E}_{c m}=\mathbf{3 0 9 6 . 8 8 0 ~ M e V} \\
\mathbf{E}=\mathbf{4 1 7 2 . 5 3 9} \mathrm{MeV}\end{array}$ \\
\hline$\frac{d \delta E}{d f_{r e v}}$ & $1.280 \mathrm{MeV} / \mathrm{Hz}$ & $0.445 \mathrm{MeV} / \mathrm{Hz}$ & $0.127 \mathrm{MeV} / \mathrm{Hz}$ \\
\hline$\frac{d \delta E}{d L}$ & $1.699 \mathrm{MeV} / \mathrm{mm}$ & $0.586 \mathrm{MeV} / \mathrm{mm}$ & $0.165 \mathrm{MeV} / \mathrm{mm}$ \\
\hline$\frac{d \delta E_{c m}}{d f_{r e v}}$ & $278.9 \mathrm{keV} / \mathrm{Hz}$ & $113.2 \mathrm{keV} / \mathrm{Hz}$ & $38.5 \mathrm{keV} / \mathrm{Hz}$ \\
\hline$\frac{d \delta E_{c m}}{d L}$ & $370.0 \mathrm{keV} / \mathrm{mm}$ & $149.3 \mathrm{keV} / \mathrm{mm}$ & $50.1 \mathrm{keV} / \mathrm{mm}$ \\
\hline
\end{tabular}

The beam revolution frequency spectrum is measured by observation of the Schottky noise of the beam on a spectrum analyzer ${ }^{1}$. The average revolution frequency of beam in the Accumulator can be measured with a precision of about 1 part in $10^{7}\left(\right.$ i.e. $\delta f_{r e v} \approx 0.1 \mathrm{~Hz}$ ). From Table $\mathrm{I}$, a $0.1 \mathrm{~Hz}$ uncertainty in $f_{\text {rev }}$ gives rise to an uncertainty in $E_{c m}$ of less than $11 \mathrm{keV}$ at the energies below the $\psi^{\prime}$.

The precision with which the orbit length is measured is difficult to estimate. A reasonable estimate is $\delta L \approx 1.2 \mathrm{~mm}$. This orbit length uncertainty yields a corresponding uncertainty in $E_{c m}$ that varies from $179 \mathrm{keV}$ at the $\psi^{\prime}$ to $60 \mathrm{keV}$ at the J/ $\psi$. The uncertainty in $E_{c m}$ due to the uncertainty in the orbit length is not negligible when compared to width of the narrow resonances in the Charmonium spectrum.

1 See: M.D. Church and J.P. Marriner, Annu. Rev. Nucl. Part. Sci. 1993, 43:253-295, section 7.

Also: C.M. Ginsberg, Spectroscopy of $\mathrm{J} / \psi$ and $\psi^{\prime}$ Charmonium Resonances, Ph.D. Thesis, Northwestern University, December 1995, sections 3.3 and 3.4 . 


\section{Measurement of the orbit length}

The measurement of the average orbit length is accomplished by measuring the orbit length difference between the orbit of interest and an orbit of known length. The orbit of known length will be hereinafter referred to as the "reference orbit."

\section{A. The Reference Orbit}

The reference orbit is obtained by decelerating antiprotons to the vicinity of a narrow, well-measured charmonium resonance. The $\mathrm{J} / \psi$ and the $\psi^{\prime}$ are ideal for this purpose. The beam is scanned in energy across the resonance and the excitation of the resonance is measured as a function of beam energy. At each step in the scan the closed orbit of the antiproton beam is measured ${ }^{2}$ and stored. An analysis of the resonance excitation data allows an accurate determination of the beam energy at each point in the scan. The orbit length associated with each of the stored orbit measurements can then be calculated by solving equation (2) for $L$. One of these orbits becomes the reference orbit, and its length becomes the reference orbit length $\left(L_{r e f}\right)$.

There are two sources of uncertainty in a value of $L_{r e f}$ that is determined in this manner: (1) the uncertainty in the published mass of the resonance ${ }^{3}$, and (2) the statistical error in the measurement of the excitation curve. The statistical error is primarily determined by the luminosity integrated at each point in the scan. The relatively large cross-sections ${ }^{4}$ for the $\mathrm{J} / \psi$ and the $\psi^{\prime}$ are sufficient to make the statistical error very small for scans of $1 \mathrm{pb}^{-1}$ or more of integrated luminosity.

The uncertainty in the length of the reference orbit $\left(\delta L_{r e f}\right)$ is related to the uncertainty in the resonance mass $\left(\delta M_{\text {res }}\right.$ ) by equation (5):

$$
\delta L_{r e f}=\frac{M_{r e s} L_{r e f}}{\gamma(p c)^{2}} \delta M_{r e s}
$$

$M_{\text {res }}$ is the resonance mass; $\gamma$ and $p$ are determined by the beam energy at the resonance peak. The $\psi$ ' mass is $M_{r e s}=3686.00 \pm 0.090 \mathrm{MeV} / \mathrm{c}^{2}$, which yields $\delta L_{r e f}=0.6 \mathrm{~mm}$. Similarly, the mass of the $\mathrm{J} / \psi$ is given by $M_{\text {res }}=3096.88 \pm 0.040 \mathrm{MeV} / \mathrm{c}^{2}$, which yields $\delta L_{r e f}=0.8 \mathrm{~mm}$.

\footnotetext{
${ }^{2}$ A measurement of the closed orbit consists of the read out of all of the beam position monitors (BPMs) in the horizontal and vertical planes.

${ }^{3}$ The resonance mass values and uncertainties used here are taken from the "Review of Particle Properties" (i.e. the Particle Data Book) prepared by the Particle Data Group (http://pdg.lbl.gov).

${ }^{4}$ The partial widths to $\bar{p}$ and the widths for inclusive $\mathrm{J} / \psi$ decay are large compared to the rest of the charmonium spectrum for the $\mathrm{J} / \psi$ and the $\psi^{\prime}$. Inclusive $\mathrm{J} / \psi$ decay (i.e. charmonium $\rightarrow J / \psi+$ anything ), where the $\mathrm{J} / \psi$ subsequently decays to $\mathrm{e}^{+} \mathrm{e}^{-}$, is a signal the E835 detector very efficiently detects.
} 
The closed orbit in the Accumulator is measured by a system of horizontal and vertical pickups (BPMs) distributed throughout the Accumulator lattice. The BPM system can accurately measure changes in horizontal or vertical beam position at the pickup locations. However, the system is not calibrated to measure the position of the beam relative to the central orbit. Thus, it is necessary to rewrite equation (8) in terms of quantities that can be accurately measured.

While it is difficult to measure the displacement of an orbit relative to the central orbit, the Accumulator BPM system is capable of accurately measuring the difference between any two closed orbits. In particular, if one of those orbits is the reference orbit we can define a $\Delta x$ and $\Delta y$ such that:

$$
\begin{aligned}
& \Delta x=x-x_{r} \\
& \Delta y=y-y_{r}
\end{aligned}
$$

$x_{r}$ and $y_{r}$ are the horizontal and vertical displacements of the reference orbit relative to the central orbit. $\Delta x$ and $\Delta y$ are the horizontal and vertical displacements of the orbit of interest from the reference orbit. given by:

In the Appendix it is shown that the first order correction to the reference orbit length is

$$
L=L_{r e f}+\tilde{N}_{x} \Delta x d s
$$

To first order, the orbit length depends only on the horizontal displacement from the reference orbit. In this approximation, distortion of vertical orbit does not affect the orbit length. 


\section{C. $\quad$ Closed Orbit Distortion Model}

The evaluation of equation (10) requires knowledge of $\Delta x$ everywhere there is non-zero curvature. In the Accumulator, the only significant curvature occurs in the main bending dipole magnets. The BPMs, from which all of our knowledge of $\Delta x$ is derived, are primarily located near the quadrupole magnets in the Accumulator lattice. What is needed then, is a model of the Accumulator horizontal orbit that relates $\Delta x$ at any location in the Accumulator lattice to the values of $\Delta x$ measured at the BPMs.

The model used for the orbit length calculation assumes that the distortion of the closed orbit from the reference orbit can be written as a superposition of dipole kicks from all of the possible sources of such a kick. The model also allows for a beam momentum error (i.e. an error that causes the beam to be radially off-center in the arcs). This superposition of kicks is written as ${ }^{6}$ :

$$
\Delta x(s)=\frac{\sqrt{\beta(s)}}{2 \sin \pi v_{x}} \sum_{i} \sqrt{\beta_{i}} \theta_{i} \cos \left(\pi v_{x}-\left|\mu(s)-\mu_{i}\right|\right)+D(s) \frac{\Delta p}{p}
$$

where:

$$
\begin{array}{ll}
\beta(s) & =\text { Horizontal beta function at longitudinal position } s \\
\beta_{i} & =\text { Horizontal beta function at the } i^{t h} \text { kicker } \\
\mu(s) & =\text { Horizontal betatron phase at } s \\
\mu_{i} & =\text { Horizontal betatron phase at the } i^{\text {th }} \text { kicker } \\
D(s) & =\text { Horizontal dispersion at } s \\
v_{x} & =\text { Horizontal betatron tune } \\
\theta_{i} & =\text { Dipole kick from the } i^{t h} \text { kicker } \\
\Delta p / p & =\text { Momentum error }
\end{array}
$$

The lattice parameters (i.e. $\beta, \mu, D$, and $v_{x}$ ) are obtained from a model of the Accumulator lattice ${ }^{7}$. The parameters of the closed orbit model are the $\theta_{i}$ and $\Delta p / p$ - these must be calculated from the BPM measurements of the Accumulator closed orbit.

The determination of the $\theta_{i}$ and $\Delta p / p$ is accomplished by substituting the BPM measurement of $\Delta \mathrm{x}$ at each horizontal BPM for $\Delta x(s)$ in equation (11) and solving for the kicks. The equation to be solved for $\theta_{i}$ and $\Delta p / p$ is:

$$
\Delta \mathbf{x}_{\mathrm{b}}=\mathbf{M}_{\mathrm{bk}} \boldsymbol{g}_{\mathbf{k}}+\mathbf{D}_{\mathrm{b}} \frac{\Delta p}{p}
$$

$\Delta \mathbf{x}_{\mathbf{b}}$ is a vector of length $N_{b}$ (where $N_{b}=$ number of horizontal BPMs) containing the BPM readout of the difference between the orbit of interest and the reference orbit, $\mathbf{D}_{\mathbf{b}}$ is a vector of

\footnotetext{
${ }^{6}$ Equation (11) must be modified for those kick elements that are so long the lattice functions vary significantly over their length (e.g. the $5^{\circ}, 10^{\circ}$, and $15^{\circ}$ horizontal bending dipoles). In this case, the summand in equation (11) must be integrated over the length of the kicker element. The contribution of these elements to the sum can be written as:

$$
\frac{\sqrt{\beta(s)}}{2 \sin \pi v_{x}} \frac{\theta_{i}}{L_{i}} \int_{s_{i}}^{s_{i}+L_{i}} \sqrt{\beta_{i}(t)} \cos \left(\pi v_{x}-\left|\mu(s)-\mu_{i}(t)\right|\right) d t
$$

${ }^{7}$ The software used for the Accumulator lattice model is the CERN product MAD (Methodical Accelerator Design). The magnetic fields and gradients required as inputs to MAD are based on magnetic measurements of Accumulator magnets at the Fermilab Magnetic Test Facility (MTF).
} 
the same length containing the value of the horizontal dispersion function at each horizontal BPM, $\boldsymbol{\theta}_{\mathbf{k}}$ is a vector of length $N_{k}$ containing the kicks from the kicker elements. $\mathbf{M}_{\mathbf{b k}}$ is the $N_{b} \times N_{k}$ matrix that connects the kicks from the kicker elements to an orbit displacement at the horizontal BPMs.

Ultimately equation (12) will be solved for $\boldsymbol{\theta}_{\mathrm{k}}$ and $\Delta p / p$. As will soon become evident, this must be handled as a least squares problem. Therefore, since not all BPMs are identical in the accuracy of their readout, each of the terms in equation (12) will be weighted by the estimated uncertainty in the BPM readout. $\Delta \mathbf{x}_{\mathrm{b}}, \mathbf{M}_{\mathbf{b k}}$, and $\mathbf{D}_{\mathbf{b}}$ become:

$$
\begin{aligned}
& b_{j} \equiv \frac{\left(\Delta x_{b}\right)_{j}}{\sigma_{j}} \\
& \left(M_{b k}\right)_{j i} \rightarrow \frac{\left(M_{b k}\right)_{j i}}{\sigma_{j}} \\
& \left(D_{b}\right)_{j} \rightarrow \frac{\left(D_{b}\right)_{j}}{\sigma_{j}}
\end{aligned}
$$

$\sigma_{j}$ is the uncertainty in the readout of the $j^{\text {th }}$ BPM. To avoid complicating the notation, the normalized and unnormalized $\mathbf{M}_{\mathbf{b k}}$ and $\mathbf{D}_{\mathbf{b}}$ bear the same name.

From equations (11) and (13), the elements of $\mathbf{M}_{\mathbf{b k}}$ are:

$$
\left(M_{b k}\right)_{j i}=\frac{\sqrt{\beta_{j} \beta_{i}}}{2 \sigma_{j} \sin \pi v_{x}} \cos \left(\pi v_{x}-\left|\mu_{j}-\mu_{i}\right|\right)
$$

It is convenient to expand the definition of $\mathbf{M}_{\mathbf{b k}}$ and $\boldsymbol{\theta}_{\mathbf{k}}$ to include the $\Delta p / p$ term. $\mathbf{M}_{\mathbf{b k}}$ is augmented by adding a column containing the vector $\mathbf{D}_{\mathbf{b}} . \Delta p / p$ is made the last element of the $\boldsymbol{\theta}$ vector. Equation (12) becomes:

$$
\begin{aligned}
\mathbf{b} & =\left(\left[\begin{array}{l}
\mathbf{M}_{\mathrm{bk}} \\
\end{array}\right]\left[\begin{array}{l}
\mathbf{D}_{\mathrm{b}} \\
\end{array}\right]\right)\left(\left[\begin{array}{c}
\boldsymbol{\theta}_{\mathbf{k}} \\
\frac{\Delta p}{p}
\end{array}\right]\right) \\
& \equiv \mathbf{M}_{\mathbf{b}} \mathbf{9}
\end{aligned}
$$

The dimensions of $\mathbf{M}_{\mathbf{b}}$ are $N_{b} \times N_{k}$ (where $N_{k}$ now is the number of kicks +1 ).

Equations (11) through (15) assume that the Accumulator lattice at the energy where the reference orbit was established is the same as the lattice at the various places in the deceleration ramps where the beam energy is to be measured. This is not always the case. What is done to account for this is to assume, that to zeroth order, the same lattice is realized at all energies of interest. The variations in the gradients of the quadrupole magnets that cause the lattice to change will also kick the beam. The kick from each quadrupole magnet is proportional to the change in normalized gradient and the displacement of the beam from the central orbit. These kicks are accommodated in the closed orbit distortion model by allowing a kick in the $\theta$ vector 
from each quadrupole. Since we are only interested in accounting for what happens to the orbit, this approach is sufficient ${ }^{8}$. Adding a kick from all of the quadrupoles has the effect of making the system in equation (15) significantly underdetermined ${ }^{9}$. The difficulties involved with solving (15) are dealt with in the next section.

Once equation (15) has been solved for $\boldsymbol{\theta}$, the displacement of the orbit relative to the reference orbit can be calculated elsewhere in the Accumulator lattice. In particular, we are now in a position to calculate $\Delta x(s)$ in the arcs so that the integral in equation (10) can be evaluated. To do this, a matrix $\mathbf{M}_{\mathbf{d k}}$ is constructed in the same manner as $\mathbf{M}_{\mathbf{b k}}$ above. $\mathbf{M}_{\mathbf{d k}}$ is the matrix that connects the kicks to horizontal distortion of the closed orbit in the main bending dipoles. The elements of $\mathbf{M}_{\mathbf{d k}}$ are given by:

$$
M_{d k}(s)_{j i}=\frac{\sqrt{\beta_{j}(s)}}{2 L_{i} \sin \pi \nu_{x}} \int_{s_{i}}^{s_{i}+L_{i}} \sqrt{\beta_{i}(t)} \cos \left(\pi v_{x}-\left|\mu_{j}(s)-\mu_{i}(t)\right|\right) d t
$$

where $M_{d k}(s)_{j i}$ connects the $i^{\text {th }}$ kick to longitudinal position $s$ in the $j^{t h}$ dipole. $L_{i}$ is the length of the $i^{\text {th }}$ kick element. The integration is necessary only when the length of the kicker element is so large that the lattice functions change substantially over its length. In a manner analogous to equation (15), $\Delta x_{d}(s)$ in the dipoles is given by:

$$
\begin{aligned}
\Delta \mathbf{x}_{\mathbf{d}}(s) & =\left(\left[\mathbf{M}_{\mathbf{d k}}(s)\right]\left[\mathbf{D}_{\mathrm{d}}(s)\right]\right)\left(\left[\begin{array}{l}
\boldsymbol{\theta}_{\mathbf{k}} \\
\frac{\Delta p}{p}
\end{array}\right]\right) \\
& \equiv \mathbf{M}_{\mathbf{d}}(s) \boldsymbol{g}
\end{aligned}
$$

$\mathbf{D}_{\mathbf{d}}(s)$ is a vector containing the value of the dispersion function at longitudinal location $s$ in the dipoles. $\mathbf{M}_{\mathbf{d}}$ is a $N_{d} \times N_{k}$ dimensional matrix, where $N_{d}$ is the number of horizontal main bending dipoles.

The integral in equation (10) becomes a sum of integrals over the main bending dipoles. Substitution of equation (17) into equation (10) gives:

$$
\begin{aligned}
L & =L_{\text {ref }}+\sum_{i=1}^{N_{d}} \int_{s_{i}}^{s_{i}+L_{4}} \kappa_{x}\left[\Delta x_{d}(s)\right]_{i} d s \\
& =L_{r e f}+\sum_{i=1}^{N_{d}} \int_{s_{i}}^{s_{i}+L_{j}} \kappa_{x}\left[\mathbf{M}_{\mathbf{d}}(s) \mathbf{g}\right]_{i} d s
\end{aligned}
$$

\footnotetext{
${ }^{8}$ This can be checked by calculating the orbit length using the both the reference orbit lattice and using the lattice where the orbit length is being measured. When this is done, the difference in the resulting orbit lengths is typically less than $0.1 \mathrm{~mm}$.

9 The number of columns of $\mathbf{M}_{\mathbf{b}}$ is the number of horizontal dipole elements (39) + the number of quadrupole magnets $(84)+1$ (for the $\Delta p / p$ term). The number of rows of $\mathbf{M}_{\mathbf{b}}$ is the number of horizontal BPMs, of which there are 48 . Thus, equation (15) represents a system of 48 equations that must be solved for 124 unknown parameters.
} 


\section{Solving for the kicks}

The next step is to solve equation (15) for $\boldsymbol{\theta}$. This requires some care for two reasons: (1) $\mathbf{M}_{\mathbf{b}}$ is generally very nearly singular ${ }^{10}$ and, (2) $\mathbf{M}_{\mathbf{b}}$ is not square. Moreover, if kicks from all of the quadrupole magnets are included, the number of columns of $\mathbf{M}_{\mathbf{b}}$ will significantly exceed the number of rows (i.e. the system is under-determined). Since $\mathbf{M}_{\mathbf{b}}$ is not square, there will not be a unique solution to equation (15). Consequently, some attention must be given to the selection of solutions that are physically meaningful.

These difficulties are overcome by manipulating the Singular Value Decomposition ${ }^{11}$ (SVD) of $\mathbf{M}_{\mathbf{b}}$. SVD reconstructs $\mathbf{M}_{\mathbf{b}}$ as the product three matrices:

$$
\mathbf{M}_{\mathbf{b}}=\mathbf{U g W g V}^{T}
$$

$\mathbf{U}$ is a $N_{b} \times N_{k}$ matrix whose columns are orthonormal, $\mathbf{W}$ is a $N_{k} \times N_{k}$ diagonal matrix with nonnegative elements, and $\mathbf{V}$ is a $N_{k} \times N_{k}$ matrix whose columns and rows are orthonormal. For reasons that will soon become apparent, the diagonal elements of $\mathbf{W}$ are called the singular values of $\mathbf{M}_{\mathbf{b}}$. These properties of $\mathbf{U}, \mathbf{W}$, and $\mathbf{V}$ can be summarized as follows:

$$
\begin{aligned}
& \mathbf{U}^{T} \mathbf{g U}=\mathbf{1} \\
& \mathbf{V}^{T} \mathbf{g V}=\mathbf{V} \mathbf{g V}^{T}=\mathbf{1} \\
& W_{i j}=\lambda_{i} \delta_{i j}
\end{aligned}
$$

The $\lambda_{i}$ 's are the singular values and $\delta_{i j}$ is the Kronecker delta. Generally, the decomposition is performed in such a way as to place the $\lambda_{i}$ 's in descending order (i.e. $\lambda_{1}>\lambda_{2}>\lambda_{3}>\ldots \lambda_{N_{k}}$ ).

If $N_{k}>N_{b}$ (i.e. if quadrupole kicks are included), $\mathbf{M}_{\mathbf{b}}$ must be augmented with $N_{k}-N_{b}$ rows of zeros to make it square. The decomposition of the augmented $\mathbf{M}_{\mathbf{b}}$ results in a $\mathbf{U}$ matrix that is $N_{k} \times N_{k}$. In this case at least the last $N_{k}-N_{b}$ diagonal elements of $\mathbf{W}$ will be zero. The SVD matrices, in this case, retain all of the properties given in (20) above.

From the properties given in (20) for the SVD matrices, an inverse of $\mathbf{M}_{\mathbf{b}}$ is given by:

$$
\begin{aligned}
\mathbf{M}_{\mathrm{n}}^{-1} & =\mathbf{V} \mathbf{g} \mathbf{W}^{-1} \mathbf{g}^{T} \\
\left(\mathbf{W}^{-1}\right)_{i j} & =\frac{\delta_{i j}}{\lambda_{i}}
\end{aligned}
$$

The problem with this particular inverse of $\mathbf{M}_{\mathbf{b}}$ is that $\mathbf{W}$ is blows up if any of the $\lambda_{i}$ 's are zero (which will always be the case when quadrupole kicks are included). Even if $\mathbf{M}_{\mathbf{b}}$ only accounts for kicks from the horizontal dipoles, it is nonetheless likely that some of the $\lambda_{i}$ 's will be very small.

The utility of singular value decomposition is that it makes provision for "factoring out" the singularity. The SVD matrices are constructed so that the columns of $\mathbf{U}$ contain an

\footnotetext{
${ }^{10} \mathbf{M}_{\mathrm{b}}$ tends to be singular because (1) a $\Delta p / p$ error and an overall bend bus error are indistinguishable, and (2) the three and six fold symmetry of the Accumulator causes some degeneracy among the rows of $\mathbf{M}_{\mathbf{b}}$.

${ }^{11}$ For an excellent overview of Singular Value Decomposition see: W. H. Press, S. A. Teukolsky, W. T. Vetterling, and B. P. Flannery, Numerical Recipes in C: The Art of Scientific Computing, Second Edition (Cambridge University Press, 1992) sections 2.6 and 15.4. The E835 off-line beam energy calculation uses a double precision version of subroutine SVDCMP taken from this book.
} 
orthonormal set of basis vectors spanning the range ${ }^{12}$ of $\mathbf{M}_{\mathbf{b}}$ and the columns of $\mathbf{V}$ contain an orthonormal set of basis vectors for the null space ${ }^{13}$ of $\mathbf{M}_{\mathbf{b}}$. Specifically, if the rank of $\mathbf{M}_{\mathbf{b}}$ is $N_{R}$, then the first $N_{R}$ values of $\lambda_{i}$ will be non-zero while the values of $\lambda_{i}$ for $N_{R}<i \leq N_{k}$ will be zero. The first $N_{R}$ columns of $\mathbf{U}$ will form an orthonormal set of basis vectors for the range of $\mathbf{M}_{\mathbf{b}}$ and columns $N_{R}+I$ through $N_{k}$ of $\mathbf{V}$ form an orthonormal set of basis vectors for the null space of $\mathbf{M}_{\mathbf{b}}$. To see how this helps, equation (15) will be recast in terms of the projections of each part on either the range or null space of $\mathbf{M}_{\mathbf{b}}$.

If $\mathbf{b}$ is not in the range of $\mathbf{M}_{\mathbf{b}}$, equation (15) cannot be solved exactly. There will be a non-zero residual vector, $\mathbf{r}$, given by:

$$
\mathbf{r}=\mathbf{M}_{\mathbf{b}} \boldsymbol{g}-\mathbf{b}
$$

In this case, the solutions of interest are those that minimize $|\mathbf{r}|$. By definition, $\mathbf{M}_{\mathbf{b}}$ can "reach" all vectors within the vector space of its range; therefore, if $\boldsymbol{\theta}$ minimizes $|\mathbf{r}|$, then $\mathbf{r}$ will not have a projection on the range of $\mathbf{M}_{\mathbf{b}}$. Writing $\mathbf{M}_{\mathbf{b}}$ in terms of its SVD matrices and multiplying through by $\mathbf{U}^{\mathrm{T}}$ gives:

$$
\mathbf{U}^{T} \mathbf{g}=\left(\mathbf{W} g \mathbf{V}^{T}\right) \mathbf{g}-\mathbf{U}^{T} \mathbf{g}
$$

In what follows, the column vector consisting of the $i^{\text {th }}$ column of any matrix $\mathbf{A}$ will be written as $\mathbf{A}^{(i)}$. It is useful to note that the vector $\mathbf{A}^{\mathrm{T}} \cdot \mathbf{v}$, where $\mathbf{v}$ is an appropriately dimensioned column vector, is given by:

$$
\left(\mathbf{A}^{T} \mathbf{g v}\right)_{i}=\sum_{k} A_{k i} v_{k}=\mathbf{v}^{T} \mathbf{g} \mathbf{A}^{(i)}
$$

That is, $\mathbf{A}^{\mathrm{T}} \cdot \mathbf{v}$ is a vector containing the inner product of $\mathbf{v}$ with each of the columns of $\mathbf{A}$. In particular, if the columns of $\mathbf{A}$ are unit vectors (as is the case with the columns of $\mathbf{U}$ and $\mathbf{V}$ ), the elements of $\mathbf{A}^{\mathrm{T}} \cdot \mathbf{v}$ will be the projection of $\mathbf{v}$ along each of the columns of $\mathbf{A}$.

Each term of equation (23) can now be written in terms of projections on the range or the null space of $\mathbf{M}_{\mathbf{b}}$.

$$
\left.\mathbf{U}^{T} \mathbf{g}=\left[\begin{array}{c}
\mathbf{0} \\
\mathbf{r}_{2}
\end{array}\right]\right\} N_{R} \quad \text { components }
$$

Since the first $N_{R}$ columns of $\mathbf{U}$ are basis vectors for the range of $\mathbf{M}_{\mathbf{b}}$, the first $N_{R}$ elements of $\mathbf{U}^{\mathrm{T}} \cdot \mathbf{r}$ are zero since $\mathbf{r}$ is a vector that does not project onto the range of $\mathbf{M}_{\mathbf{b}} \cdot \mathbf{r}_{2}$ is a vector that lies completely outside the range of $\mathbf{M}_{\mathbf{b}}$. The matrix of singular values looks like:

$$
\mathbf{W}=\left(\begin{array}{cc}
\Lambda & 0 \\
\mathbf{0} & \mathbf{0}
\end{array}\right), \quad \boldsymbol{\Lambda}=\left(\begin{array}{ccc}
\lambda_{1} & & 0 \\
& 0 & \\
0 & & \lambda_{N_{R}}
\end{array}\right)
$$

$\Lambda$ is the $N_{R} \times N_{R}$ diagonal matrix containing all of the non-zero singular values. The first term on the right hand side of equation (23) becomes:

\footnotetext{
${ }^{12}$ The range of a matrix $\mathbf{A}$ is the vector space of all vectors $\mathbf{b}$ for which $\mathbf{A} \cdot \mathbf{x}=\mathbf{b}$. The dimension of this space is the rank of $\mathrm{A}$.

${ }^{13}$ The null space of a matrix $\mathbf{A}$ is the vector space of all vectors $\mathbf{x}$ for which $\mathbf{A} \cdot \mathbf{x}=\mathbf{0}$. The dimension of this space is the nullity of $\mathbf{A}$.
} 


$$
\begin{aligned}
& \mathbf{V}^{T} \boldsymbol{g}=\left[\begin{array}{c}
\boldsymbol{\theta}_{1} \\
\boldsymbol{\theta}_{\text {null }}
\end{array}\right] \begin{array}{ll}
N_{R} & \text { components } \\
\}_{N_{k}}-N_{R} & \text { components }
\end{array} \\
& \left.\mathbf{W g V}^{T} \boldsymbol{g}=\left[\begin{array}{cc}
\boldsymbol{\Lambda} \boldsymbol{\theta}_{1} \\
\mathbf{0}
\end{array}\right]\right\} \begin{array}{ll}
\} N_{R} & \text { components } \\
N_{k}-N_{R} & \text { components }
\end{array}
\end{aligned}
$$

$\boldsymbol{\theta}_{1}$ is the component of $\boldsymbol{\theta}$ that lies outside of the null space of $\mathbf{M}_{\mathrm{b}}$. Since the last $N_{k}-N_{R}$ columns of $\mathbf{V}$ form a basis for the null space of $\mathbf{M}_{\mathbf{b}}$, these elements of $\mathbf{V}^{T} \boldsymbol{\theta}$ will give the projection of $\boldsymbol{\theta}$ on the null space, $\boldsymbol{\theta}_{\text {null }}$.

The last term in equation (23) is:

$$
\mathbf{U}^{T} \mathbf{g}=\left[\begin{array}{l}
\mathbf{b}_{r} \\
\mathbf{b}_{2}
\end{array}\right] \begin{array}{ll}
\} N_{R} & \text { components } \\
N_{b}-N_{R} & \text { components }
\end{array}
$$

As in equation (25), $\mathbf{b}_{r}$ is the projection of $\mathbf{b}$ on the range of $\mathbf{M}_{\mathbf{b}}$. $\mathbf{b}_{2}$ is the component of $\mathbf{b}$ that lies outside the range of $\mathbf{M}_{\mathbf{b}}$.

Substituting (23), (27), and (28) into (23) gives:

$$
\begin{aligned}
{\left[\begin{array}{c}
\mathbf{0} \\
\mathbf{r}_{2}
\end{array}\right] } & =\left[\begin{array}{c}
\boldsymbol{\Lambda} \boldsymbol{g}_{1} \\
\mathbf{0}
\end{array}\right]-\left[\begin{array}{l}
\mathbf{b}_{r} \\
\mathbf{b}_{2}
\end{array}\right] \\
\boldsymbol{\Lambda} \mathbf{g}_{1} & =\mathbf{b}_{r} \\
\mathbf{r}_{2} & =-\mathbf{b}_{2}
\end{aligned}
$$

Equation (29) shows that the residual is entirely due to $\mathbf{b}_{2}$. Note also that the $N_{k}-N_{R}$ components of $\boldsymbol{\theta}_{\text {null }}$ are completely arbitrary in that they do not contribute to the residual vector. Thus, there is a family of solutions of equation (15) that minimize the residual. We will have to further distinguish among this family of solutions to get the $\boldsymbol{\theta}$ that is to be used in the closed orbit distortion model.

From equations (27), (28), and (29), the solutions to equation (22) that minimize the residual are given by:

$$
\begin{aligned}
\boldsymbol{\theta} & \left.=\mathbf{V} \oiint \begin{array}{c}
\boldsymbol{\theta}_{1} \\
\boldsymbol{\theta}_{\text {null }}
\end{array}\right] \\
& =\mathbf{V}\left[\begin{array}{c}
\boldsymbol{\Lambda}^{-1} \mathbf{g U}_{R}^{T} \mathbf{g} \mathbf{b} \\
\boldsymbol{\theta}_{\text {null }}
\end{array}\right]
\end{aligned}
$$

where $\mathbf{U}_{R}$ is the $N_{b} \times N_{R}$ matrix that consists of the first $N_{R}$ columns of $\mathbf{U}$. This gives a solution for $\theta$ that minimizes the residual and isolates that part of the solution that does not contribute to the residual.

$$
\boldsymbol{\theta}=\mathbf{V}_{R} \mathbf{g} \Lambda^{-1} \mathbf{g} \mathbf{U}_{R}^{T} \mathbf{g b}+\mathbf{V}_{N} \boldsymbol{g}_{\text {sull }}
$$

where $\mathbf{V}_{R}$ is the $N_{k} \times N_{R}$ matrix containing the first $N_{R}$ columns of $\mathbf{V}$, and $\mathbf{V}_{N}$ is the $N_{k} \times\left(N_{k}-N_{R}\right)$ matrix that consists of the last $N_{k}-N_{R}$ columns of $\mathbf{V}$. 
The singularity of $\mathbf{M}_{\mathbf{b}}$ gives rise to the second term of equation (31). The first term of equation (31) can be written in terms of the original SVD matrices if the inverse of $\mathbf{W}$ is redefined as follows:

$$
W_{i}^{-1} \equiv \begin{cases}\lambda_{i} & i \leq N_{R} \\ 0 & i>N_{R}\end{cases}
$$

With this definition, the first term of equation (31) is:

$$
\boldsymbol{\theta}=\left(\mathrm{VgW}^{-1} \mathrm{gU}^{T}\right) \mathbf{g b}
$$

The expression in parentheses appears to be identical to what was initially identified as $\mathbf{M}_{\mathbf{b}}{ }^{-1}$ in equation (21). Two things have changed since then: (1) the re-definition of $\mathbf{W}^{-1}$ in equation (32) and, (2) the isolation of the singular term in equation (31).

If $\mathbf{M}_{\mathbf{b}}$ were not singular, the solution of equation (15) is unique and is given by equation (33). If $\mathbf{M}_{\mathbf{b}}$ is singular, equation (33) is still a particular solution, however the general solution now contains an arbitrary contribution from the null space - hence the second term in equation (31). An important feature of the SVD solution is that equation (33) gives the particular solution that minimizes the length of $\theta$. It is evident from equation (31) that the singular term can only increase $|\boldsymbol{\theta}|$.

The length of $\theta$ is proportional to the rms kick from all of the kick elements. These kicks are expected to be small. There are several reasons to look for a small $\boldsymbol{\theta}$. First, the Accumulator orbit is corrected to be as close as possible to the reference orbit ${ }^{14}$. Second, the physical aperture of the Accumulator places a significant constraint on the magnitude of any individual kick. Finally, the dipole and quadrupole magnets and their associated power supplies are limited as to the magnitude of the kick they can give the beam. Therefore, the $\boldsymbol{\theta}$ chosen for the orbit length calculation is the particular solution of $\mathbf{b}=\mathbf{M}_{\mathbf{b}} \cdot \boldsymbol{\theta}$ that minimizes $|\boldsymbol{\theta}|-$ i.e. the solution given by equation (33).

One remaining issue must be taken up before leaving the subject of Singular Value Decomposition. This issue has to do with the proper handling of very small singular values. Computationally the difference between zero and non-zero is not as clear-cut as it is analytically. Very small singular values are indicative of a matrix that is badly conditioned. The most likely consequence of small $\lambda_{i}$ is the presence of large positive and negative elements in $\theta$ that are ordered in such way that their individual effects on $\mathbf{b}$ very nearly cancel against each other. The solution to this problem is to establish a singular value threshold. The SVD threshold is implemented by another modification to the definition of $\mathbf{W}^{-1} . \mathbf{W}^{-1}$ is now given by:

$$
W_{i}^{-1}= \begin{cases}\frac{1}{\lambda_{t}} & \lambda_{i}>\lambda_{\text {thresh }} \\ 0 & \lambda_{i} \leq \lambda_{\text {thresh }}\end{cases}
$$

$\lambda_{\text {thresh }}$ is the SVD threshold value.

What must be settled now is where to set $\lambda_{\text {thresh }}$. This can be studied by examining the behavior of $\boldsymbol{\theta}$ and $|\mathbf{r}|$ as a function of the threshold setting. The value of $i$ in equation (34) that corresponds to the last non-zero $1 / \lambda_{i}$ is called the threshold index. The variation of $\theta$ with the

\footnotetext{
${ }^{14}$ Generally, the deviation from the reference orbit is less than $5 \mathrm{~mm}$.
} 
threshold index, $\boldsymbol{\theta}(i)$, can be explicitly seen by writing equation (33) in terms of inner products with columns of $\mathbf{V}$ and $\mathbf{U}$. The resulting expression is:

$$
\left.\boldsymbol{\theta}(i)=\sum_{k=1}^{i} \mathbf{V}^{(k)} \mathfrak{g} \frac{\mathbf{U}^{(k)} \mathbf{g} \mathbf{b}}{\lambda_{k}}\right)
$$

The rms kick, $\theta_{r m s}(i)$ and the variation of $|\mathbf{r}|$ with the threshold index are then given by:

$$
\begin{aligned}
\theta_{r m s}(i) & =\frac{\left|\boldsymbol{\theta}^{(i)}\right|}{\sqrt{N_{k}}} \\
|\mathbf{r}(i)| & =\left|\mathbf{b}-\mathbf{M}_{\mathbf{b}} \mathbf{g} \boldsymbol{\theta}(i)\right|
\end{aligned}
$$

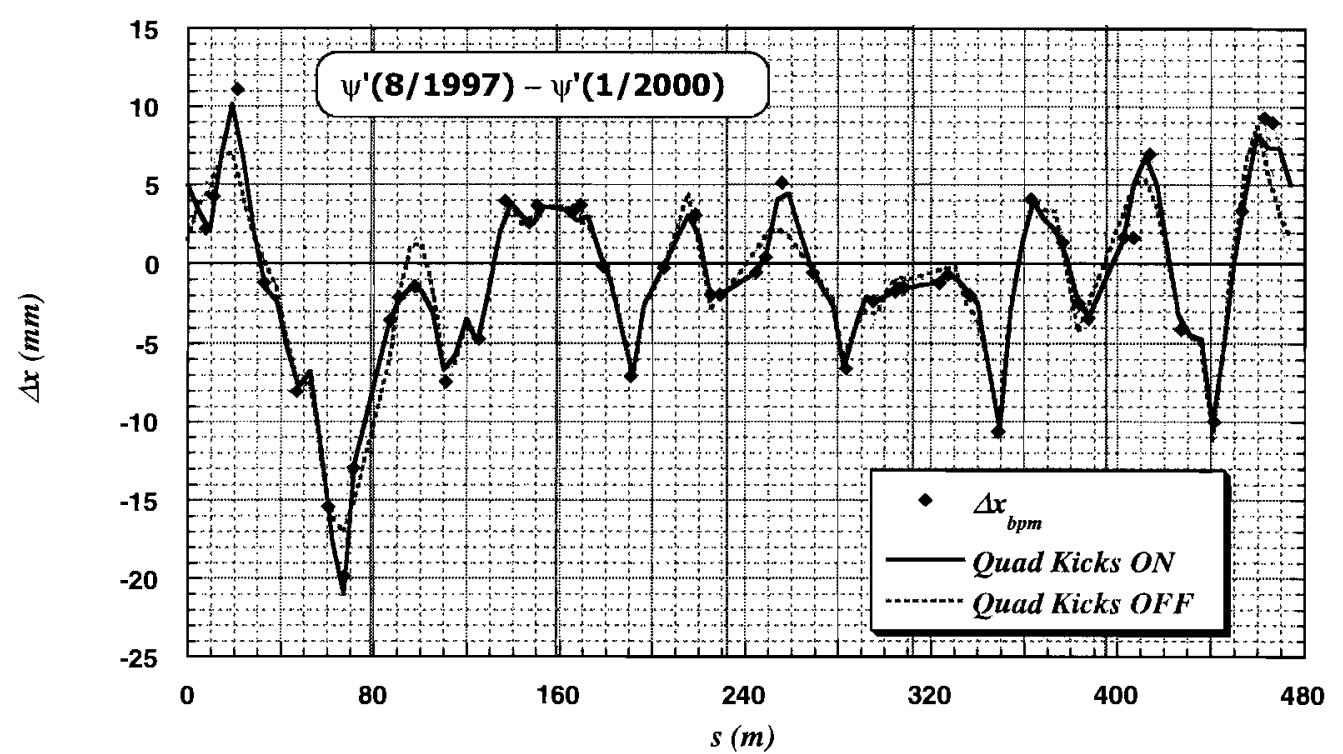

Figure 2. This figure shows the difference between the horizontal orbit during the $1997 \psi^{\prime}$ scan and the horizontal orbit during the February $2000 \psi^{\prime}$ scan. The $\Delta x$ plotted here is given by: $\Delta x=x\left(\psi^{\prime} 1997\right)-x\left(\psi^{\prime} 2000\right)$. The horizontal axis, $s$, is the longitudinal position in the Accumulator. The black diamonds are BPM measurements; the blue line represents the closed orbit distortion model with quadrupole kicks, the red dashed line is the orbit model without quadrupole kicks. The orbit difference seen here is much larger than what is encountered during normal running.

Figure 3 and Figure 4 illustrate the information required to determine the value of $\lambda_{\text {thresh }}$. These graphs show the dependence of $\theta_{r m s}(i),|\mathbf{r}(i)|$, and $\Delta L(i)$ on the SVD threshold setting. The reference orbit for this analysis shown in these figures is from the January 2000 scan of the $\psi$ '. The test orbit is from the August 1997 scan of the $\psi$ '. These orbits are useful because: (1) the length of each is known ${ }^{15}$, (2) the August, 1997 Accumulator lattice at the $\psi$ ' energy was quite different from the January 2000 lattice ${ }^{96}$, and (3) the orbits themselves are significantly different from one another (see Figure 2). The use of these orbits represents a very severe test of the calculation. Figure 3 shows the SVD threshold dependence when quadrupole kicks are included while Figure 4 gives the situation when only horizontal dipole kicks are considered.

\footnotetext{
${ }^{15}$ The length of the $\psi^{\prime}(8 / 1997)$ orbit is $474.04965 \mathrm{~m}$. The length of the $\psi^{\prime}(1 / 2000)$ orbit is $474.05403 \mathrm{~m}$.

${ }^{16}$ A modification of the Accumulator lattice to raise the value of $\gamma_{t}$ (for stochastic cooling purposes) had taken place between these two measurements.
} 


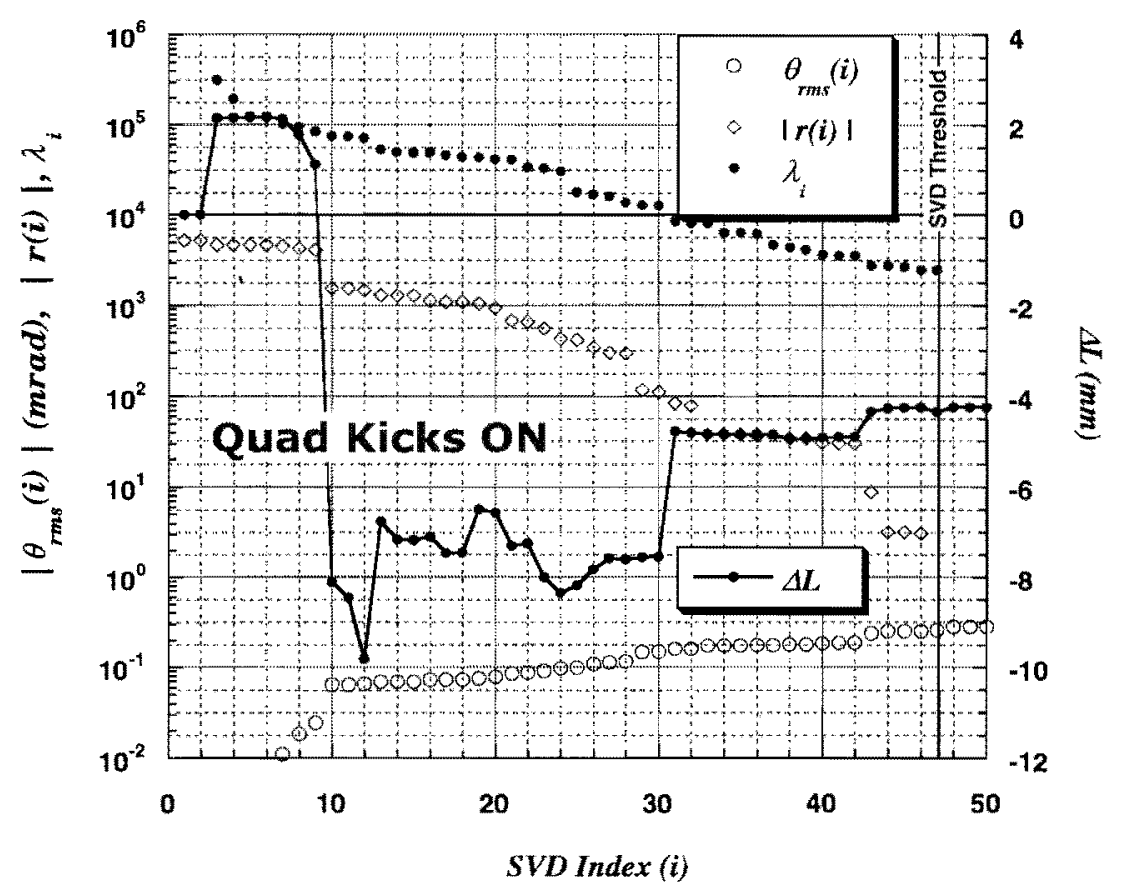

Figure 3. $\theta_{r m s},|\mathbf{r}|$ and $\Delta L$ are calculated with different SVD thresholds. This calculation includes kicks from all of the quadrupoles. The horizontal axis gives the threshold index, $i$. The singular values for $i>48\left(=N_{b}\right)$ are zero. The vertical red line is the index corresponding to the SVD threshold used for the orbit length calculation. There is one non-zero singular value below the threshold $\left(\lambda_{48}=2.5 \times 10^{-7}\right)$. For $i>46,|\mathbf{r}|<10^{-4}$. The reference orbit for this analysis is from the January 2000 scan of the $\psi^{\prime}$. The test orbit is from the August 1997 scan of the $\psi^{*}$.

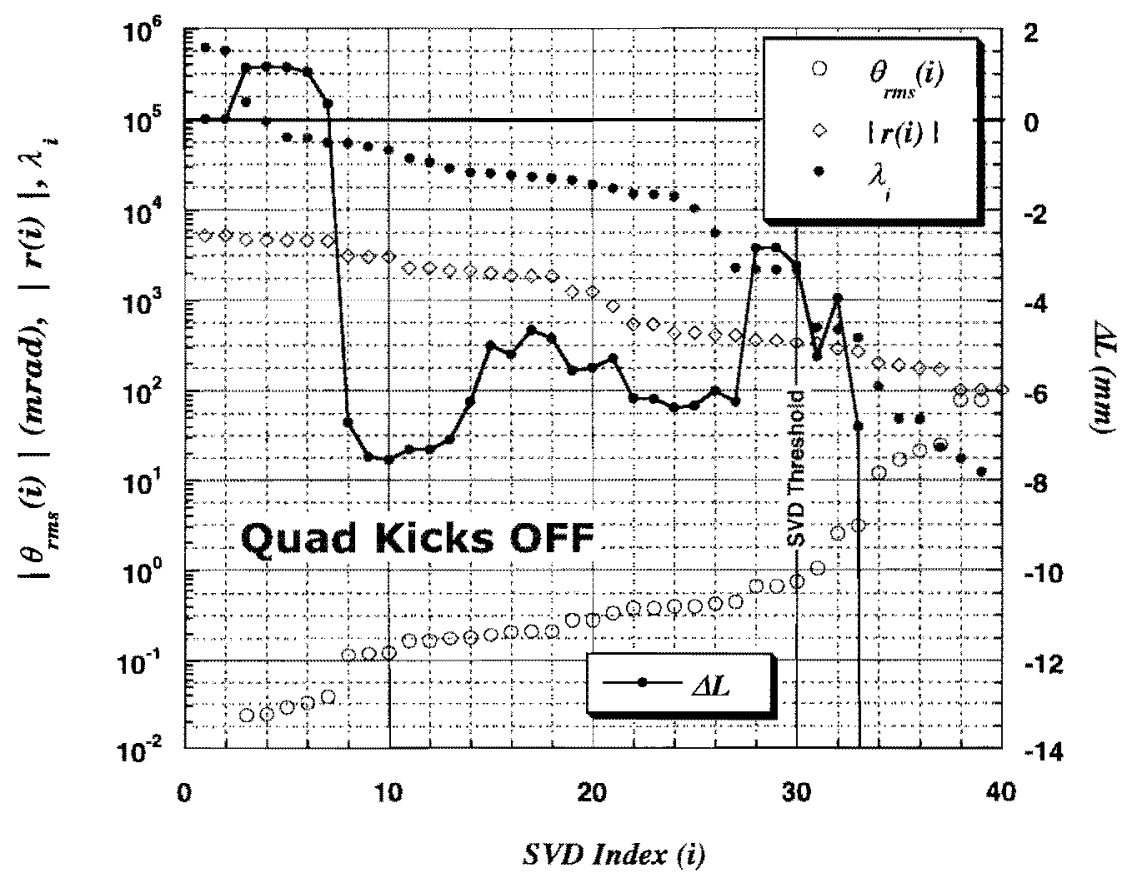

Figure 4. $\theta_{r m s},|\mathbf{r}|$ and $\Delta L$ are calculated with different SVD thresholds. This calculation includes only kicks from the dipole elements in the Accumulator $\left(N_{k}=40\right)$. All 40 singular values are non-zero. The reference orbit for this analysis is from the January 2000 scan of the ' $\psi^{\prime}$. The test orbit is from the August 1997 scan of the $\psi^{\prime}$. 
What one would like to see is the situation in Figure 3 . This figure shows the results of the orbit length calculation where $\boldsymbol{\theta}$ includes kicks from all of the quadrupoles. The index corresponding to the singular value threshold was set at $i=47$. In the vicinity of this threshold setting, observe the following: (1) $|\mathbf{r}|$ is very small, (2) $\theta_{r m s}$ is small and not increasing as the threshold is lowered, (3) there is a large (10 order of magnitude) drop in $\lambda$, and most importantly, (4) the value of $\Delta L$ is relatively insensitive to where the threshold is $\operatorname{set}^{17}$. In this case, the setting of the singular value threshold is unambiguous.

A much less desirable situation is illustrated in Figure 4 . Here, $\boldsymbol{\theta}$ only contains kicks from the horizontal dipoles. In this case, it is difficult to determine where the singular value threshold should be set. In contrast to the calculation with the quadrupole kicks included, Figure 4 exhibits the following: (1) $|\mathbf{r}|$ is relatively large and does not decrease appreciably as the threshold is lowered, (2) $\theta_{r m s}$ is increasing rapidly as the threshold is lowered, (3) the singular values (except $\lambda_{40}$ ) are not small, and finally, (4) $\Delta L$ is very sensitive to where the threshold is set.

There are two conclusions from this analysis of Figure 3 and Figure 4: (1) Kicks from the quadrupole magnets should be included when the lattice upon which the reference orbit was measured and that upon which the test orbit was measured differ significantly. (2) Having done (1), the setting of the singular value threshold is unambiguous.

As a second example and also to document the situation on the present deceleration ramp, Figure 6 and Figure 7 show the same two analyses for an orbit measured during a scan of the $\chi_{1}$ resonance in February 2000. The reference orbit used is the January $2000 \psi^{\prime}$ reference orbit. The Accumulator lattice at the $\chi_{1}$ is not the same as at the $\psi^{\prime}$, however, the difference is much less than that between $\psi^{\prime}(1997)$ and $\psi^{\prime}(2000)$.

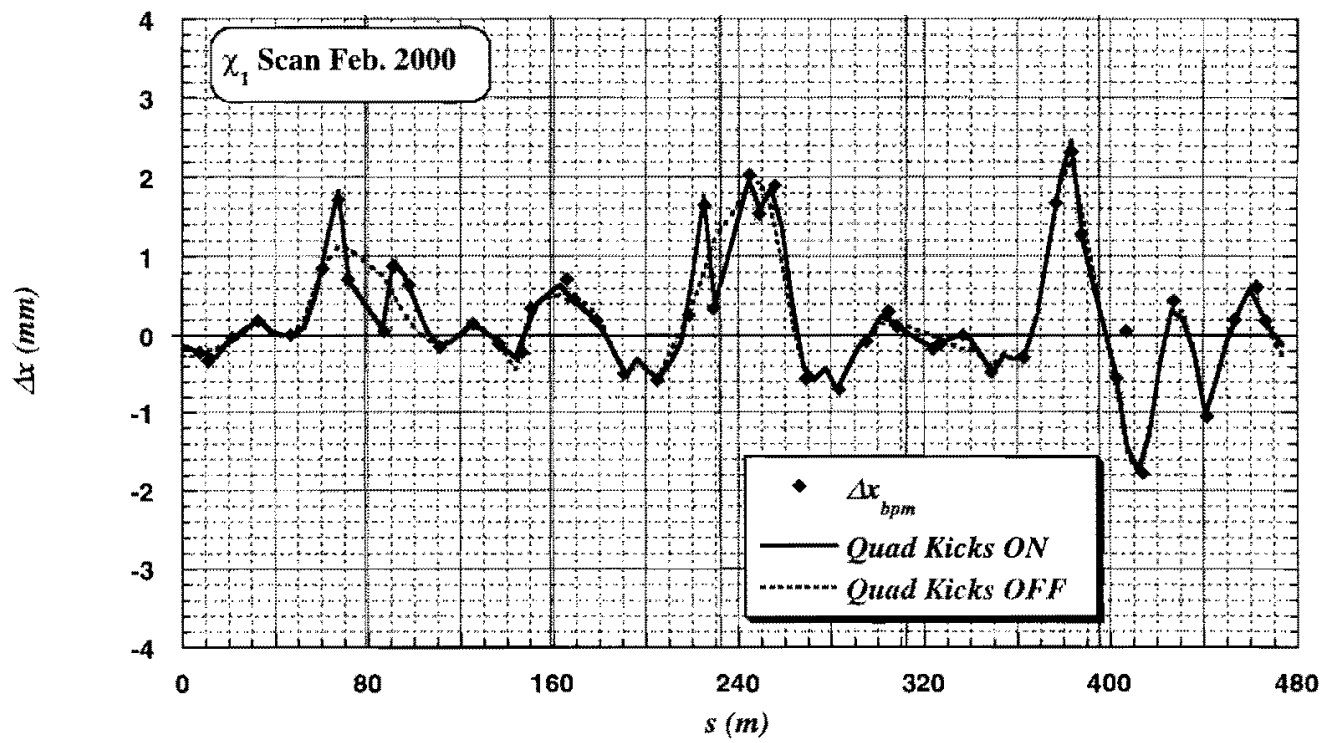

Figure 5. Horizontal difference orbit during the February, 2000 scan of the $\chi_{1}$. The $\Delta x$ plotted here is given by: $\Delta x=x\left(\chi_{1}\right)-x\left(\psi^{3}\right)$.

Figure 5 shows the horizontal difference orbit (relative to the $\psi$ ' reference orbit) during the February 2000 scan of the $\chi_{1}$ resonance. Figure 6 and Figure 7 show the variation of $\theta_{\text {rms }}(i)$,

${ }^{17}$ The value of the orbit length measured using the SVD threshold setting indicated in Figure 3 was 0.05 mm different from the orbit length established for this orbit during the August, $1997 \psi^{\prime}$ scan. 
$|\mathbf{r}(i)|$, and $\angle L(i)$ with the SVD threshold setting. The analysis of Figure 6 includes quadrupole kicks; the analysis of Figure 7 does not.

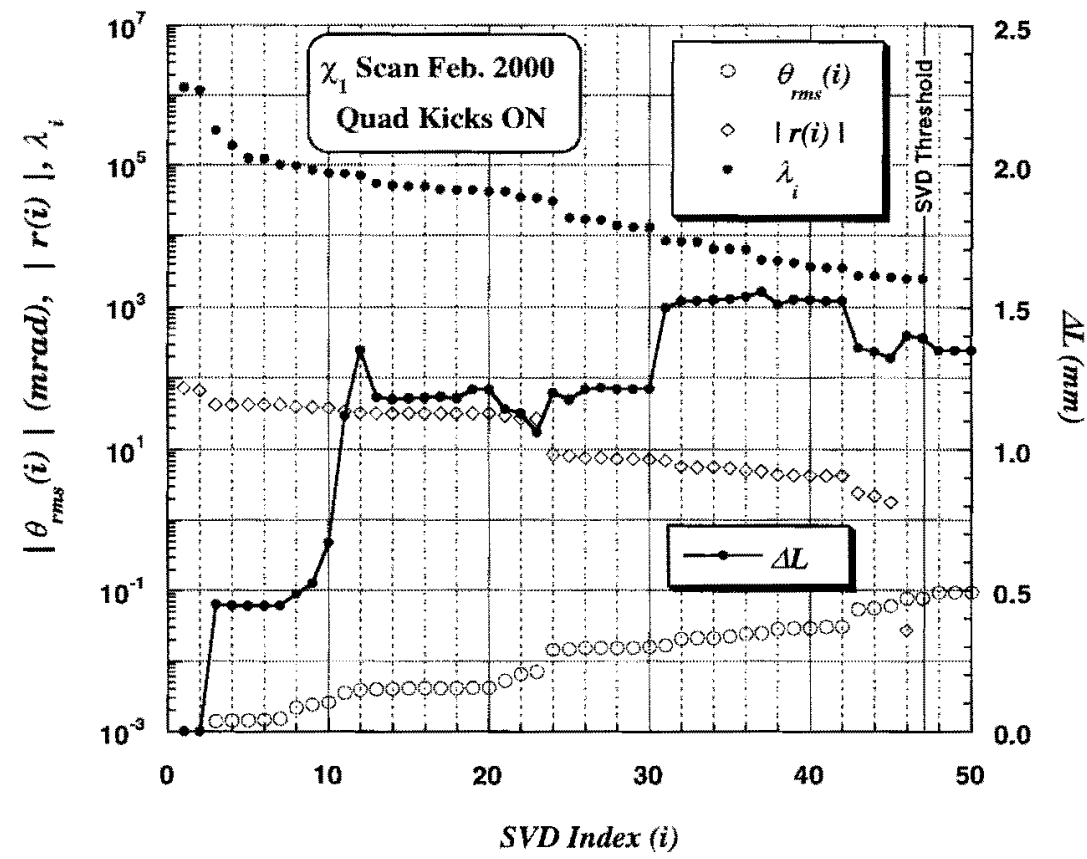

Figure 6. $\theta_{r m s},|\mathbf{r}|$ and $\Delta L$ are calculated with different SVD thresholds. This calculation includes kicks from all of the quadrupoles.

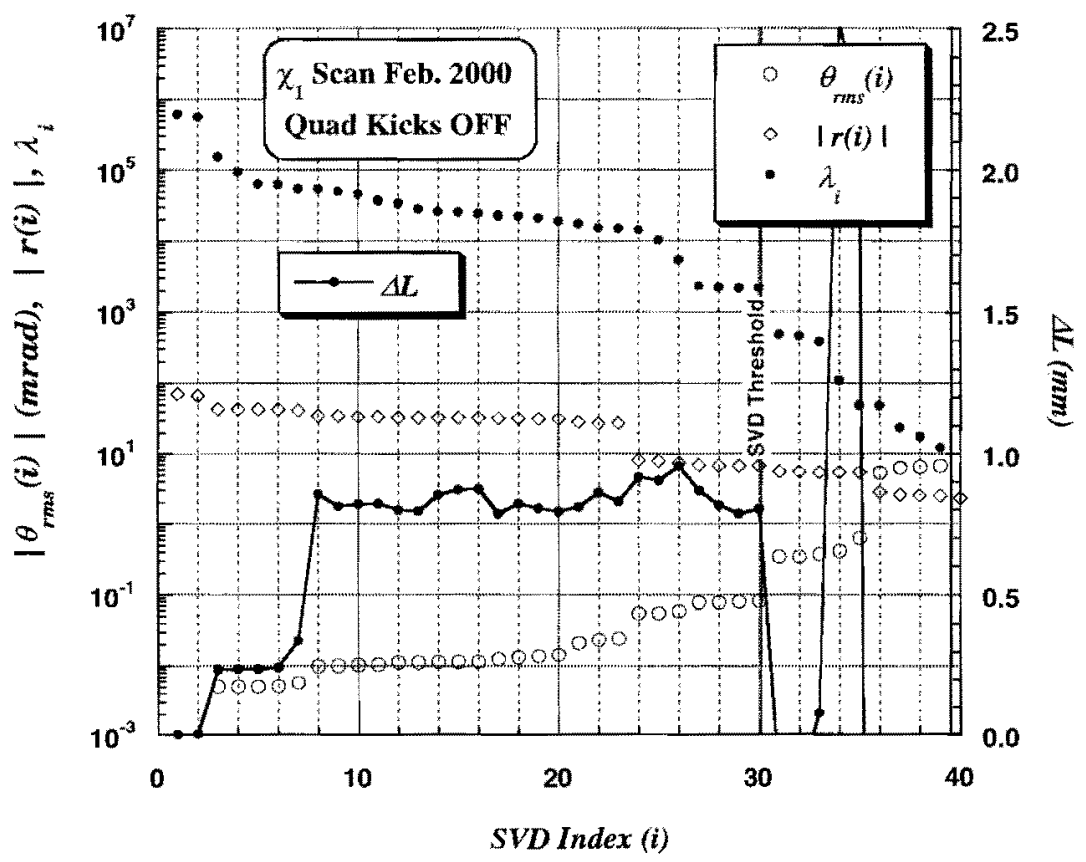

Figure 7. $\theta_{r m s},|\mathbf{r}|$ and $\Delta L$ are calculated with different SVD thresholds. This calculation includes only kicks from the dipole elements in the Accumulator $\left(N_{k}=40\right)$.

As was the case with the analysis of Figure 3 and Figure 4, the stability of the solution is much greater if kicks from the quadrupoles are allowed. The kicks calculated the dipoles and quadrupoles from the analysis associated with Figure 6 is shown in Figure 8. The analysis that 
includes quadrupole kicks results in an orbit length of $474.05543 \mathrm{~m}(1.39 \mathrm{~mm}$ greater than the length of the reference orbit). The analysis that excludes quadruple kicks results in an orbit length of $474.05484 \mathrm{~m}(0.80 \mathrm{~mm}$ greater than the length of the reference orbit).

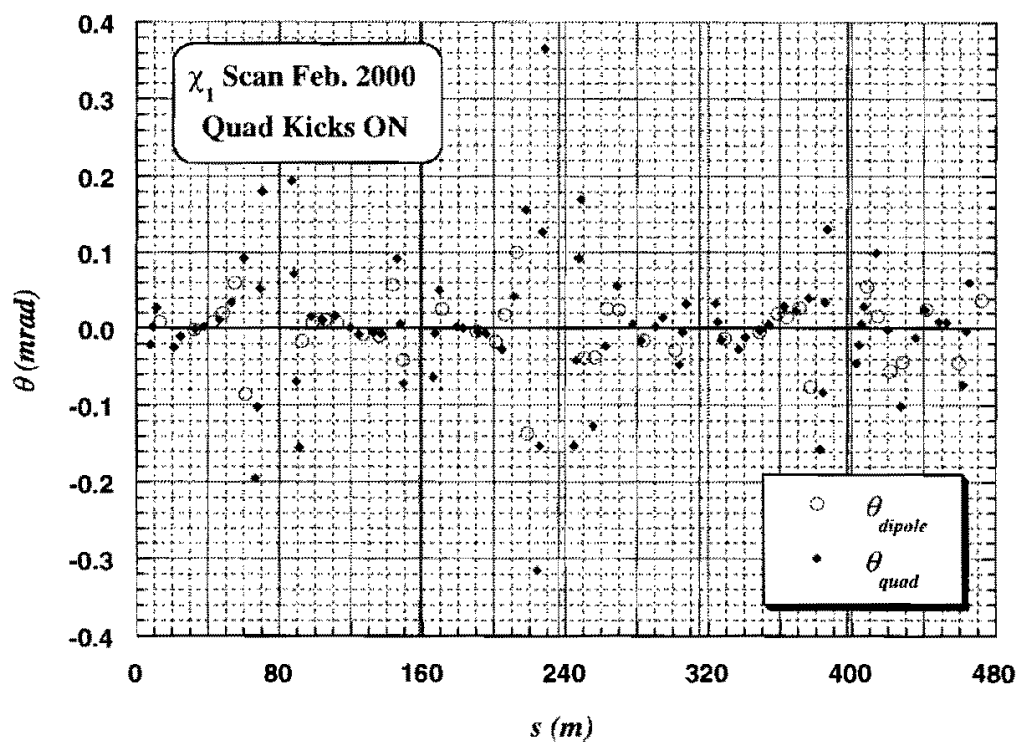

Figure 8. Kicks calculated from the $\Delta x$ shown in Figure 5 and the analysis of Figure 6.

\section{E. BPM Constants}

Since the matrix $\mathbf{M}_{\mathbf{b}}$ depends only on the Accumulator lattice and the BPM weights ( $\sigma_{j}$ in equation (13)), the SVD analysis need only be performed once ${ }^{18}$. All of the information contained in $\mathbf{M}_{\mathbf{b}}$ can be compressed into a vector, $\mathbf{C}$, of $N_{b}$ BPM constants such that equation (18) can be written as:

$$
L=L_{r e f}+\mathbf{C g} \Delta \mathbf{x}_{\mathbf{b}}
$$

From equation (18), $\mathbf{C g} \Delta \mathbf{x}_{\mathbf{b}}$ is given by:

$$
\begin{aligned}
\mathbf{C g} \Delta \mathbf{x}_{\mathbf{b}} & =\sum_{i=1}^{N_{d}} \int_{s_{i}}^{s_{i}+L_{i}} \kappa_{x}\left[\mathbf{M}_{\mathbf{d}}(s) \mathbf{g}\right]_{i} d s \\
& =\sum_{i=1}^{N_{d}} \int_{s_{i}}^{s_{i}+L_{i}} \kappa_{x}\left[\sum_{j=1}^{N_{k}} M_{d_{i j}}(s) \theta_{j}\right] d s \\
& =\sum_{i=1}^{N_{d}} \int_{s_{i}}^{s_{i}+L_{i}} \kappa_{x}\left[\sum_{j=1}^{N_{k}} M_{d_{i j}}(s) \theta_{j}\right] d s \\
& =\sum_{i=1}^{N_{d}} \int_{s_{i}}^{s_{i}+L_{i}} \kappa_{x}\left[\sum_{j=1}^{N_{k}} M_{d_{i j}}(s)\left(\mathbf{V} \mathbf{g W}^{-1} \mathbf{g U}^{T} \mathbf{g}\right)_{j}\right] d s
\end{aligned}
$$

\footnotetext{
${ }^{18}$ If a horizontal BPM fails or its readout becomes unreliable, it is masked out of the calculation by setting its $\sigma$ to a very large number. Since this changes $\mathbf{M}_{\mathbf{b}}$, the SVD analysis must be performed again.
} 
The last line follows from equation (33). For purposes of simplifying the notation somewhat, define the matrix $M_{i j}$ as:

$$
M_{i j}=\int_{s_{i}}^{s_{i}+L_{i}} M_{d_{i j}}(s) d s
$$

Putting this into equation (38) yields:

$$
\begin{aligned}
\mathbf{C g} \Delta \mathbf{x}_{\mathbf{b}} & =\kappa_{x} \sum_{i=1}^{N_{k}} \sum_{j=1}^{N_{k}} M_{i j}\left(\mathbf{V} \mathbf{g W}^{-1} \mathbf{g U}^{T} \mathbf{g b}\right)_{j} \\
& =\kappa_{x} \sum_{i=1}^{N_{t}} \sum_{j=1}^{N_{k}} \sum_{k=1}^{N_{h}} M_{i j}\left(\mathbf{V} \mathbf{g} \mathbf{W}^{-1} \mathbf{g U}^{T}\right)_{j k} \frac{\Delta x_{b_{k}}}{\sigma_{k}} \\
& =\kappa_{x} \sum_{i=1}^{N_{j}} \sum_{j=1}^{N_{k}} \sum_{k=1}^{N_{k}} \sum_{n=1}^{N_{k h}} M_{i j} \frac{V_{j n} U_{k n}}{\lambda_{n}} \frac{\Delta x_{b_{k}}}{\sigma_{k}}
\end{aligned}
$$

$N_{t h r}$ is the index of the smallest singular value above threshold ${ }^{19}$. The BPM constants - i.e. elements of $\mathbf{C}$, are:

$$
C_{k}=\frac{\kappa_{x}}{\sigma_{k}} \sum_{i=1}^{N_{i}} \sum_{j=1}^{N_{k}} \sum_{n=1}^{N_{k n}} M_{i j} \frac{V_{j n} U_{k n}}{\lambda_{n}}
$$

\section{A Test of the Beam Energy Calculation}

The best test of the orbit length calculation is to evaluate its ability to properly determine the length of an orbit of known length. This test has been done using the reference orbit for the 1997 E835 run as the test orbit. This is the orbit measured during the August 1997 scan of the $\psi^{\prime}$, described earlier. An analysis of the orbit length calculation for this orbit is given above in section II.D (see Figure 2, Figure 3, and Figure 4). This is a very rigorous test of the orbit length calculation since this orbit is significantly different from the present reference orbit (see Figure 2) and the Accumulator lattice at that time was different from the present lattice.

In 1997 , the length of this orbit was determined to be $474.04965 \mathrm{~m} \pm 0.60 \pm 0.07 \mathrm{~mm}$. The first uncertainty quoted is derived from the uncertainty in the published mass of the $\psi^{\prime}$ (cf. Equation (6)). The second uncertainty is from the statistical uncertainty in the determination of the beam energy when the orbit was measured. The statistical uncertainty is the uncertainty in the determination of $\psi^{\prime}$ mass from the resonance excitation measured during the scan.

The length of this orbit using the January, $2000 \psi$ ' reference orbit is $474.04970 \mathrm{~m}^{20}$. This is just $0.05 \mathrm{~mm}$ different from the length determined in 1997. This measurement is well within the uncertainty in the length of this orbit as determined from the resonance scan.

\footnotetext{
${ }^{19}$ Recall that the $\lambda$ 's occur in descending order.

${ }^{20}$ The orbit length calculation producing this result included quadrupole kicks.
} 


\section{Appendix: Derivation of Equation (10)}

\section{Separating out the length of the reference orbit}

The expression for the orbit length, written in terms of the reference orbit and the horizontal and vertical displacements from it, is:

$$
L=\sqrt{1+2 \kappa_{x}\left(x_{r}+\Delta x\right)+\kappa_{x}^{2}\left(x_{r}+\Delta x\right)^{2}+\left(x_{r}^{\prime}+\Delta x^{\prime}\right)^{2}+\left(y_{r}^{\prime}+\Delta y^{\prime}\right)^{2}} d s
$$

Rearranging things a little gives:

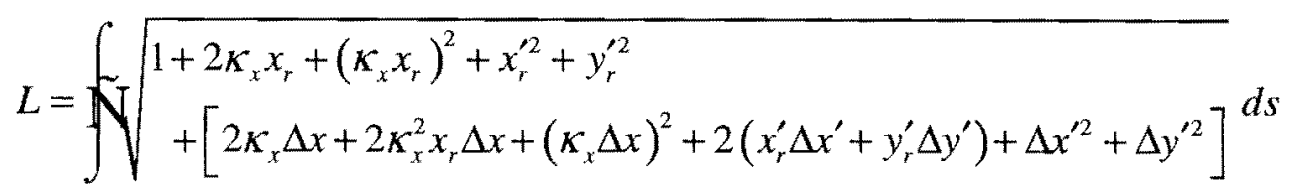

Note that if the terms in the square brackets were zero, the length in equation (A-2) reduces to the length of the reference orbit, $L_{r e f}$. To simplify the next part of the calculation, the terms in the square brackets will be collectively designated $\delta F\left(x_{r}, x_{r}^{\prime}, y_{r}^{\prime} ; \Delta x, \Delta x^{\prime}, \Delta y^{\prime}\right)$ and the terms having to do with the length of the reference orbit will be designated $\delta F_{r}\left(x_{r}, x_{r}, y_{r}{ }^{\prime}\right)$. Equation (A-2) becomes:

$$
L=\widehat{N} \sqrt{1+\delta F_{r}+\delta F} d s
$$

where,

$$
\begin{array}{ll}
\delta F_{r}\left(x_{r}, x_{r}^{\prime}, y_{r}^{\prime}\right) & =2 \kappa_{x} x_{r}+\kappa_{x}^{2} x_{r}^{2}+x_{r}^{\prime 2}+y_{r}^{2} \\
\delta F\left(x_{r}, x_{r}^{\prime}, y_{r}^{\prime} ; \Delta x, \Delta x^{\prime}, \Delta y^{\prime}\right) & =2 \kappa_{x} \Delta x+2 \kappa_{x}^{2} x_{r} \Delta x+\kappa_{x}^{2} \Delta x^{2}+2\left(x_{r}^{\prime} \Delta x^{\prime}+y_{r}^{\prime} \Delta y^{\prime}\right)+\Delta x^{\prime 2}+\Delta y^{\prime 2}
\end{array}
$$

The procedure now is to expand the integrand of equation (A-3) in a power series in $\delta F_{r}$ and $\delta F$. This is valid if $\delta F_{r}+\delta F<1$; a condition that is certain to be true ${ }^{1}$. Two applications of the binomial theorem to the integrand of $(A-3)$ gives:

$$
\begin{aligned}
\sqrt{1+\delta F_{r}+\delta F} & =\sum_{n=0}^{\infty}\left(\begin{array}{c}
1 / 2 \\
n
\end{array}\right)\left(\delta F_{r}+\delta F\right)^{n} \\
& =1+\sum_{n=1}^{\infty}\left(\begin{array}{l}
1 / 2 \\
n
\end{array}\right) \sum_{m=0}^{n}\left(\begin{array}{l}
n \\
m
\end{array}\right) \delta F_{r}^{n-m} \delta F^{m} \\
& =1+\sum_{n=1}^{\infty}\left(\begin{array}{c}
1 / 2 \\
n
\end{array}\right)\left[\delta F_{r}^{n}+\sum_{m=1}^{n}\left(\begin{array}{l}
n \\
m
\end{array}\right) \delta F_{r}^{n-m} \delta F^{m}\right]
\end{aligned}
$$

\footnotetext{
$1 x$ and $y$ are typically less than $10 \mathrm{~mm} . \rho_{x}=17.46 \mathrm{~m}$ in the arcs of the Accumulator. Therefore, $\kappa_{x} x<\frac{10 \mathrm{~mm}}{17.46 \mathrm{~m}}=5.9 \times 10^{-4}$. The derivatives of $\mathrm{x}$ and $\mathrm{y}$ are typically less than $1 \mathrm{~mm} / \mathrm{m}\left(\right.$ i.e. $\left.x^{\prime}, y^{\prime}<1 \times 10^{-3}\right)$
} 
continuing:

$$
\begin{aligned}
\sqrt{1+\delta F_{r}+\delta F} & =\sum_{n=0}^{\infty}\left(\begin{array}{l}
1 / 2 \\
n
\end{array}\right) \delta F_{r}^{n}+\sum_{n=1}^{\infty} \sum_{m=1}^{n}\left(\begin{array}{c}
1 / 2 \\
n
\end{array}\right)\left(\begin{array}{l}
n \\
m
\end{array}\right) \delta F_{r}^{n-m} \delta F^{m} \\
& =\sqrt{1+\delta F_{r}}+\sum_{n=1}^{\infty} \sum_{m=1}^{n}\left(\begin{array}{c}
1 / 2 \\
n
\end{array}\right)\left(\begin{array}{l}
n \\
m
\end{array}\right) \delta F_{r}^{n-m} \delta F^{m}
\end{aligned}
$$

The integration of the first term of the final expression of (A-6) is just the length of the reference orbit. Substitution of this result into equation (A-3) gives:

$$
L=L_{r e f}+\mathcal{f}\left[\sum_{n=1}^{\infty} \sum_{m=1}^{n}\left(\begin{array}{c}
1 / 2 \\
n
\end{array}\right)\left(\begin{array}{l}
n \\
m
\end{array}\right) \delta F_{r}^{n-m} \delta F^{m}\right] d s
$$

This equation is exact. The problem has been reduced to a calculation of a correction to be applied to the length of the reference orbit.

\section{Identifying the important terms}

Ultimately, we're interested in calculating the first order correction to $L_{\text {ref. }}$. For the time being, all terms up to second order will be retained. Table A-1 gives those terms of equation (A-7) with $n, m \leq 2$ up to $2^{\text {nd }}$ order.

Table A-1. Orbit Length Expansion Terms to $2^{\text {nd }}$ Order

\begin{tabular}{|l|l|r|l|}
\hline$n$ & $m$ & $\left(\begin{array}{c}1 / 2 \\
n\end{array}\right)\left(\begin{array}{c}n \\
m\end{array}\right)$ & \multicolumn{1}{|c|}{$\delta F_{r}^{n-m} \delta F^{m}$} \\
\hline 1 & 1 & $\frac{1}{2}$ & $2 \kappa_{x} \Delta x+2 \kappa_{x}^{2} x_{r} \Delta x+\kappa_{x}^{2} \Delta x^{2}+2\left(x_{r}^{\prime} \Delta x^{\prime}+y_{r}^{\prime} \Delta y^{\prime}\right)+\Delta x^{\prime 2}+\Delta y^{\prime 2}$ \\
\hline 2 & 1 & $-\frac{1}{4}$ & $4 \kappa_{x}^{2} x_{r} \Delta x$ \\
\hline 2 & 2 & $-\frac{1}{8}$ & $4 \kappa_{x}^{2} \Delta x^{2}$ \\
\hline
\end{tabular}

With all second order and lower terms explicitly included, equation (A-7) becomes:

$$
L=L_{r e f}+f\left(\kappa_{x} \Delta x+x_{r}^{\prime} \Delta x^{\prime}+y_{r}^{\prime} \Delta y^{\prime}+\frac{\Delta x^{\prime 2}+\Delta y^{\prime 2}}{2}+\star(3)\right) d s
$$

The only first order term in this expression is the first term in the integrand. Therefore, to first order, the orbit length is given by:

$$
L=L_{r e f}+\mathcal{N}_{x} \Delta x d s
$$

